\title{
La actualidad decantada. Enfoques y estilos en los titulares de portada
}

\author{
Dolors PALAU SAMPIO \\ Universitat de València \\ dolors.palau@uv.es
}

Recibido: $17 / 02 / 2013$

Aceptado: 09/09/2013

\begin{abstract}
Resumen
La portada constituye un escaparate de los contenidos del periódico, un espacio de identidad y, desde hace unos años, cada vez más, un elemento de marketing, un reclamo para despertar el interés del lector. A partir del estudio comparativo de un centenar de portadas de diez periódicos españoles, el artículo analiza el enfoque que ofrecen sobre una decena de temas de gran interés informativo. La combinación de las aportaciones de la teoría del framing y de una metodología pragmaestilística permite captar con mayor nitidez el matiz, el punto de vista empleado para definir unos hechos informativos, presentar a los agentes que intervienen o apuntar unas causas o posibles consecuencias. En definitiva, perfilar mejor el estudio de los encuadres de la realidad social que ofrecen los medios.
\end{abstract}

Palabras clave: prensa, portada, teoría del framing, pragmaestilística, punto de vista.

\section{The actuality decanted. Frames and styles in the front page titles}

\begin{abstract}
The front page constitutes a showcase of the contents of the newspaper, a space of identity and for years now, increasingly, an element of marketing, a tool to attract the reader. Based on a comparative study from a hundred cover pages of ten newspapers, our article analyses the focus on ten topics of great interest published by them. The contributions of the framing theory in a combination with a pragmastylistic methodology make possible discover more clearly the nuances, and also the point of view used to define some news topics, to present the agents that participate or to point to the causes or possible consequences. In short, the mutual assistance between them defines better the analysis of the frames from the social reality that offer the mass media.
\end{abstract}

Keywords: press, front page, framing theory, pragmastylistics, media viewpoint

\section{Referencia normalizada}

PALAU SAMPIO, Dolors (2013): "La actualidad decantada. Enfoques y estilos en los titulares de portada”. Estudios sobre el Mensaje Periodístico. Vol. 19, Núm. 2 (julio-diciembre), págs.: 825-839. Madrid, Servicio de Publicaciones de la Universidad Complutense.

Sumario: 1. Introducción. El poso de la actualidad en primera página. 2. Un escaparate privilegiado. Evolución y factores de influencia. 3. Marco teórico y metodología. 4. Enfoques sobre diez temas relevantes. 5. Conclusiones. 6. Referencias.

\section{Introducción. El poso de la actualidad en primera página}

El diccionario de la Real Academia Española (RAE) ofrece cuatro acepciones del verbo decantar, que apelan a dos ideas generales: al proceso de separación de un líquido del poso que contiene y a la acción de "inclinarse, tomar partido o decidirse". Ambas opciones responden, en términos metafóricos o convencionales, a la operación que realizan las portadas respecto al contenido general de los periódicos. La primera página constituye, en este sentido, la esencia decantada de una edición informativa: en ella se vierte el poso de las varias decenas de hojas que la integran; $y$, al mismo tiempo -o precisamente por esta necesidad de síntesis-, la posibilidad de 
matiz se traduce en aristas más afiladas. La interpretación de la realidad se resuelve en una inclinación más evidente, más nítida, buscando también la complicidad en los ojos del lector que se acerque al quiosco.

Más allá de las líneas editoriales, los periódicos coinciden en la selección de temas de portada cuando estos presentan una significación excepcional, es decir, cuando reúnen los ingredientes de noticiabilidad necesarios para figurar en la carta de asuntos preferentes. En estos casos, la elección conlleva también, casi de manera directa, la ubicación en un lugar relevante y la concesión de un espacio generoso. Sin embargo, la concordancia a la hora de destacar como relevante un hecho noticioso choca a menudo con las interpretaciones que se hacen del mismo, de modo que una lectura comparada muestra a menudo versiones casi contradictorias. Es en este punto, en esta interpretación de segundo nivel, propiamente textual, en la que centrará su atención este artículo. Para ello toma como base las aportaciones de la teoría del framing, que han demostrado su validez a la hora de identificar los elementos en los que se manifiesta el encuadre o el enfoque, y las de la pragmaestilística, que completan esta aproximación con una observación más precisa sobre las opciones lingüísticas empleadas en la narración informativa.

Los resultados que aquí se presentan son fruto de una investigación realizada sobre la base de un centenar de portadas publicadas en diez periódicos españoles. En todas ellas se han analizado los elementos de titulación referidos al tema principal, a partir de una selección de entre los más relevantes de 2012.

\section{Un escaparate privilegiado. Evolución y factores de influencia}

Pese a su importancia estratégica como escaparate de contenidos y transmisor de la esencia informativa del medio, no abundan los estudios dedicados a analizar las portadas de la prensa, especialmente en el ámbito español. Los que han centrado su atención en este apartado lo han hecho en dos aspectos que destacan su transformación en elemento de marketing y captación de lector: los cambios en el diseño, por una parte, y la selección de temas y la creciente homogeneización de contenidos, por otra.

Las metáforas asociadas a la portada de los periódicos son múltiples. En unos casos se habla de vitrina, en otros de escaparate, de expositor o de ventana, pero siempre se incide en la idea de exhibición, de presentación pública, y por tanto, de atracción, de seducción para despertar la atención -y la compra- del lector. K. Barnhurst y J. Nerone (2001: 189-190) asimilan el periódico a una especie de grandes almacenes. "Both offered a range of goods and services to a range of consumers. Both displayed their goods: the front page had become a crowded store window". Los autores, en un estudio que analiza la evolución del diseño de las portadas de los periódicos entre 1885 y 1985 -desde la época victoriana hasta la eclosión de la ola de rediseños que personalizó USA Today-, subrayan el papel central que ha adquirido esta página:

The face of the newspaper was now the front page. The design of the front page became the crucial feature in the form of the newspaper. There a newspaper announced its identity with a distinctive nameplate and showcased its content. During the ensuing century $[\ldots]$, the front page would represent the serious intentions of the newspaper [...] was thought to signal the real function of the paper (Barnhurst y Nerone, 2001: 190). 
Barnhurst y Nerone (2001: 214) sostienen que los cambios en el diseño de las portadas tienen más de evolución cultural que de revolucionaria innovación tecnológica El proceso fraguado a lo largo del pasado siglo, con evidentes puntos de conexión con otros ámbitos artísticos, cuajó en un estilo moderno en el que simplicidad, orden y autoridad acabaron por ganar la batalla. Y junto a estos valores, una idea de profesionalismo que influyó de forma determinante en el paso de unas portadas que semejaban una densa jungla - con noticias y anuncios mezclados sin orden ni concierto- al esfuerzo por trazar en estas páginas en una especie de mapa del mundo:

Mapping is also a visual activity, and newspaper front pages became defined in the professional lore as primarily visual [...]. The number of illustrations increased, and more of them stood alone; especially as the century wore on, information became packaged in graphic form [...]. The visual (as well as the textual) presentation of news demanded order, hierarchy, and usability (Barnhurst y Nerone, 2001: 216).

Las portadas racionalizaron su espacio y se convirtieron en la brújula a través de la cual los periodistas digerían y organizaban las noticias para sus lectores. Esta tarea se evidenció con la creciente presencia en portada de noticias firmadas por los redactores de la casa -especialmente a partir de los años 60 (Barnhurst y Nerone 2001: 216)-. No constituye un asunto menor el hecho de que esta página tenga la misión de cartografiar o mapear los temas más importantes de la jornada ${ }^{1}$. En ella se establece el orden de prioridades para el lector, de acuerdo con el filtro de cada medio. El problema, como sugiere P. J. Boczkowski (2010: 16-19), surge cuando la homogeneización trasciende el diseño y penetra en la selección de temas. Pese a que estas cuestiones cuentan con una tradición de varias décadas de análisis, el autor echa en falta una mayor integración entre los estudios que han puesto la atención en las prácticas de imitación entre periodistas, por una parte, y los que, por otra, se han centrado en el carácter homogéneo de la información, asumidos de manera separada por investigadores de distinto perfil. Tras analizar diversas ediciones de los dos principales periódicos impresos argentinos - $\mathrm{Cla}$ rín y La Nación- y de sus versiones online, en este caso junto al competidor Infobae.com, el autor extrae cuatro conclusiones:

First is an increase in the similarity in the selection of news and its front-page presentation for print newspapers that coincides with the timing of the growth in the volume and frequency of online news publishing. Second is a high level of similarity in print and online outlets in the contemporary scenario. Third is a high level of similarity in the narration of stories for online sites. Fourth, these patterns apply only to hard news (Boczkowski, 2010: 84).

En estas coincidencias influyen de forma importante tanto factores organizacionales como de carácter económico. Boczkowski (2010: 59-60) apela a dos dinámicas clave en la producción informativa: el seguimiento de otros medios $\left(\right.$ monitoring $\left.^{2}\right)$ y la

1 "Domesticar el siempre caótico mundo de lo noticiable (que el diario pretende 'anunciar' en tapa al límite de sus posibilidades de espacio)", como lo denomina R. Biselli (2005: 105-106).

2 El seguimiento en las rutinas productivas, a través, no ya del cara a cara, sino de la mediación tecnológica. 
imitación. Su balance señala que estas prácticas están vinculadas a un tipo de contenido, a las llamadas noticias duras (2010: 60).

Las páginas externas del periódico, la primera y la última, constituyen, según $\mathrm{M}$. Mouillaud (2002: 101), un espacio sensible, "uma membrana do jornal, que é sua interface com o mundo exterior". R. Biselli (2005: 105) considera parciales los intentos de responder a cuestiones como: “QQué relación establecen las primeras páginas de los diarios con sus lectores? ¿De qué manera [...] un diario configura una imagen de sí en un vínculo indisoluble con la postulación de determinadas modalidades de lectura?". $\mathrm{Su}$ crítica se centra "fundamentalmente en quienes restringen el papel de las portadas de los diarios a exclusivas apuestas ideológicas, políticas y/o comerciales". No se trata, dice, de negarlos, sino "de entrever sus riesgos de reduccionismo y el enmascaramiento que impondría a las múltiples estrategias discursivas actuantes en un dispositivo más complejo de lo que generalmente se cree". La portada constituye un signo de identidad clara e inapelable, que trata de imponer y consolidar entre los lectores "una imagen de sí" (Biselli, 2005: 105). El diario sensacionalista no se esconde, se reivindica en la primera página, como lo hace la llamada prensa seria (Mouillaud, 2002: 32).

En las últimas décadas los periódicos han experimentado lo que se define como un proceso de tabloidización, que se manifiesta tanto en aspectos formales como de contenido, tanto en la presentación, en el diseño, como inevitablemente en aquello que se cuenta (Conboy, 2006). El proceso que envuelve la conversión del formato sábana broadsheet, asociado a la quality press- a tabloide se refleja también en ciertos cambios en la composición de la portada: "Fewer items, sometimes just one (especially in the case of The Independent), shorter and more emphatic headlines, making it look like a magazine cover, and more oriented to the newspaper's proposal than to the main news of the day" (Coperías y Gómez Mompart, 2013).

A pesar de los cambios en el formato y del terreno que han ido conquistando los contenidos de interés humano y el llamado infoentertainment en la prensa de prestigio, distintos analistas señalan que este hecho no ha repercutido en la misma medida a la hora de seleccionar los temas de portada -aunque sí es perceptible su influencia en la redacción- ${ }^{3}$. En las conclusiones de su estudio sobre la prensa francesa, N. Hubé asegura:

Le changement des «Unes» dans le sens d'une vitrine chargée d'attirer le lecteur se traduit par l'éviction des éditoriaux de la première page et plus généralement d'un abaissement des positionnements politiques trop marqués. De manière plus surprenante, lorsqu'on s'intéresse au contenu des «Unes», ce qui peut s'apparenter à une «tabloizidation» de la forme ne conduit pas à une «tabloizidation» du choix des sujets. En effet, il ressort de nos comptages que l'actualité traitée reste massivement politique [...]. La part de l'information internationale s'est certes très fortement réduite, mais au profit de l'information politique nationale et des pages de «société» qui consistent pour beaucoup en un traitement des politiques publiques, en d'autres termes de la politique par un autre moyen (Hubé, 2010: 265).

3 "Notons aussi que, à côté du choix des sujets, l'écriture des titres est effectivement plus orientée vers des logiques d'audience en traitant plus aisément le jeu politique" (Hubé, 2010: 266). 
Los resultados pueden hacerse extensibles a ámbitos culturales muy distintos, como es el caso de la prensa india. A partir de un análisis de las portadas de los cuatro principales diarios publicados en inglés, sus autores observan que los temas que mayor peso específico tienen en estas páginas son los de política (entre un 34 y un $48 \%$ ), aunque "the political coverage over years assumed more and more new dimensions with the increasing politicization of more and more spheres along with the increasing globalization and privatization" (Murthy et al., 2010: 50).

Hubé recalca que, desde la década de los 90 -como consecuencia de la perenne crisis de la prensa diaria-, la portada se ha convertido en un instrumento de marketing, $\mathrm{y}$ apunta a cómo la idea ha permeabilizado el trabajo periodístico y ha favorecido el protagonismo de los responsables de gestión y diseño sobre la redacción. Esta tendencia ha desviado la atención del ciudadano-lector al lector-consumidor (Hubé, 2010: 254).

En paralelo se importa un discurso que va cuajando, el que vincula modernización con racionalización: "À la «rationalisation» de la forme correspond aussi un processus de rationalisation des modes de production du quotidien" (Hubé, 2010: 256). Se impone la identidad gráfica como "imagen de marca", difundida a través de campañas de fidelización, así como la idea de que "La forme c'est le fond" (Hubé, 2010: 261). En definitiva, la fuerte competencia y la caída de suscripciones -que obliga a convencer a diario al lector- han impulsado rediseños cada vez más inspirados en la lógica del marketing, con títulos breves e ilustraciones más ostensibles, cambios que han transformado las portadas en un señuelo comercial (Hubé, 2010: 269).

\section{Marco teórico y metodología}

La denominada teoría del framing, del encuadre, del enfoque o de los marcos se ha convertido en un método recurrente a la hora de abordar el tratamiento informativo de los medios, especialmente en las tres últimas décadas. Sin embargo, como han señalado distintos autores, está lejos de consolidarse como un modelo sólido -R. Entman (1993: 51-58) habla de paradigma fracturado- o, como indica D. Scheufele (1999: 103-122), de contar con una definición sistemática, capaz de integrar aproximaciones atomísticas en una teoría sólida. C. Vreese (2005: 51-62), por su parte, advierte que su creciente popularidad ha ido de la mano de una significativa inconsistencia en su aplicación. En el ámbito español, también M. L. Humanes y J. J. Igartua (2004: 202) han incidido en estos aspectos: "El concepto de encuadre es el ejemplo perfecto de la carencia de una explicación clara, precisa y operativa, que también sufren otros muchos conceptos en la investigación en comunicación".

Si la teoría de la agenda-setting centra su atención en la selección mediática, es decir, en la influencia de los medios a la hora de poner sobre la mesa un conjunto de prioridades, de temas que merecen atención ${ }^{4}$, la teoría del framing -attribute agenda setting o segundo nivel de la agenda setting- tiene como objetivo evaluar los ele-

4 Esta teoría tiene como fundamento el estudio realizado por M. Mccombs y D. Shaw (1972: 176-187) sobre la campaña de las elecciones presidenciales de EEUU de 1968, que permitió constatar la influencia de los medios a la hora de transferir la relevancia de una noticia desde su agenda a la de la opinión pública. 
mentos de énfasis que intervienen a la hora de seleccionar y presentar una noticia. Como recoge S. Ghanem (1997: 156) -a partir de aportaciones como la de M. Mccombs-, los marcos de los medios pueden dividirse en cuatro dimensiones: subtemas, mecanismos que encuadran (extensión y ubicación), atributos cognoscitivos y atributos afectivos. Estas dos últimas variables se refieren, por una parte, a la forma en que los medios definen los temas -por ejemplo, en términos de conflicto- y, por otra -teniendo en cuenta que las noticias comunican mucho más que simplemente hechos-, al tono en que se transmiten, que se expresa a través de la estructura narrativa de la noticia y busca la respuesta emocional del público (Ghanem, 1997: 158).

Las investigaciones sobre la segunda dimensión de la agenda, indican Humanes e Igartua (2004: 207-208), amplían el foco de atención a "qué atributos son más relevantes en la cobertura de los acontecimientos". Este efecto se conoce como priming y se refiere "al impacto que la agenda puede tener sobre las evaluaciones de los individuos respecto a personas y temas".

La teoría del framing tiene su origen en el desarrollo de la sociología interpretativa, en tres escuelas cuyos autores, como dice M. T. Sádaba (2001: 148), "irán sembrando el terreno que permita la aparición del framing como concepto explicativo en las ciencias sociales": el interaccionismo de la Escuela de Chicago, la fenomenología y la etnometodología. En el contexto de la sociología interpretativa, la teoría del framing se convierte en el instrumento que permite describir el proceso de interpretación y significaciones particulares para acceder a la realidad (Sádaba 2001: 148). La traslación del concepto desarrollado por G. Bateson en el campo de la psicología a la sociología y al estudio de los medios llegó de la mano E. Goffman y su obra Frame Analysis. An essay on the Organization of Experience $(1974,2006)$. En ella presentó el marco o frame como "el sistema de premisas, de instrucciones necesarias para descifrar, para dar sentido al flujo de acontecimientos. El frame permite definir las situaciones de interacción y la competencia de los individuos interactuantes. Además el marco implica expectativas de tipo normativo" (Rodrigo, 2001: 173).

La introducción de lo social a la comprensión del framing -una perspectiva influyente tanto en su aplicación al campo periodístico como al de los movimientos sociales- vino de la mano Goffman (Sádaba, 2001: 152). El estudio del encuadre desde la teoría de la comunicación se desarrolló en las décadas de los 70 y 80 , como una crítica a las pretensiones objetivistas (2001: 158). De este modo, indica Sádaba (2001: 159), "la respuesta que ofrece la teoría del framing al objetivismo es negar sus postulados, ya que argumenta que, cuando cuenta lo que sucede, el periodista encuadra la realidad y aporta su punto de vista". Entman explica que esta posibilidad se sustenta en dos operaciones:

Framing essentially involves selection and salience. To frame is to select some aspects of a perceived reality and make them more salient in a communicating text, in such a way as to promote a particular problem definition, causal interpretation, moral evaluation, and/or treatment recommendation for the item described (Entman, 1993: 52).

En definitiva, consciente o inconscientemente, periodistas y medios realizan "framing judgments in deciding what to say, guided by frames (often called schemata) that 
organize their belief systems". El texto, afirma, contiene estos frames, que ejercen su influencia a cuatro niveles:

define problems -determine what a causal agent is doing with what costs and benefits, usually measured in terms of common cultural values; diagnose causes- identify the forces creating the problem; make moral judgments -evaluate causal agents and their effects; and suggest remedies- offer and justify treatments for the problems and predict their likely effects (Entman, 1993: 52).

Ante la posibilidad de establecer un análisis más allá del texto, Entman (1993: 52) destaca que los frames "have at least four locations in the communication process: the communicator, the text, the receiver, and the culture". S. Kim, D. Scheufele y J. Shanahan (2002: 21) sugieren que el concepto de framing "implies that the way a given piece of information is described creates different outcomes among audiences". Como recogen Humanes e Igartua (2004: 209), el framing "asume que la cobertura de los medios tiene un efecto en la audiencia no a través de los temas o aspectos de un tema, sino que son las diferencias terminológicas o semánticas en la descripción de un tema las que evocan respuestas en la audiencia".

Si bien la teoría del framing arroja luz sobre el encuadre desde el que se informa de determinados temas, es interesante completar su contribución con la que puede ofrecer un análisis más detallado de los aspectos lingüísticos que intervienen en el proceso. Esta investigación apuesta una metodología pragmático-estilística (Palau, 2008) que haga posible, a través de las marcas lingüísticas observables y analizables, descubrir el punto de vista del periodista en el texto, en definitiva, su modo de configurar una realidad que contribuye a designar y crear frente a propuestas objetivistas.

El análisis del estilo en los textos periodísticos -o, en este caso, en los elementos de titulación- debe situarse en el terreno de una pragmática que contemple los factores lingüísticos y extralingüísticos que han intervenido en su producción. De aquí la elección de la pragmaestilística, la vertiente de estudio del estilo -heredera de la tradición estilística- en el marco de la lengua en uso, siguiendo a L Hickey (1987), como método adecuado para la investigación.

El estudio centra la atención en los cuatro niveles que delimitan el encuadre: la definición del problema, el diagnóstico de las causas, la evaluación de los agentes y de las consecuencias, y la sugerencia de remedios o soluciones. Ello implica identificar cuáles son las principales estrategias que se ponen en marcha a la hora de exponer de forma breve y concisa -como exigen las limitaciones de espacio de los titulares-, los hechos relevantes. Es decir, entre las diferentes tareas realizadas por el periodista (designar, avanzar en la información, destacar o introducir otras voces) y la manifestación de éstas en el texto, su expresión a través de una serie de operadores lingüísticos, como se detalla a continuación.

\section{Enfoques sobre diez temas relevantes}

Las portadas de la prensa española analizadas responden a la regla de imitación aludida por Boczkowski: todas ellas se hacen eco de asuntos que, tanto por la notoriedad de sus protagonistas o su trascendencia, quedarían clasificados en el grupo de hard 
news. Los valores noticiosos a la hora de diseñar la agenda-setting son, en este sentido, comunes. Sin embargo, esta coincidencia en la selección -en el ¿qué es noticia?no se reproduce en lo que Boczkowski llama la mirada, la singular perspectiva bajo la que se ofrecen las noticias, como factor distintivo respecto a la competencia y a otros actores del mismo paisaje mediático.

El corpus de análisis está compuesto por cien portadas de diez periódicos españoles de información general, que responden a distintas líneas editoriales y ámbitos de cobertura, publicadas en Madrid (El País [EP], El Mundo [EM], ABC [ABC], La Razón [LR], La Gaceta [LG]); Barcelona (La Vanguardia [LV], El Periódico de Catalunya [EpC]); Valencia (Levante-EMV [LE]); Bilbao (El Correo [EC]); y A Coruña (La Voz de Galicia [LVG]). La selección reúne desde temas políticos (accidente del Rey en una cacería, dimisión de Esperanza Aguirre, elecciones vascas y gallegas, y elecciones catalanas ${ }^{5}$ ), a otros económicos (dimisión de Rodrigo Rato, rescate a la banca e incremento de la prima de riesgo ${ }^{6}$ ), económico-sociales (huelga del 29-M ${ }^{7}$ ) o de sucesos-tribunales (sentencia al juez Baltasar Garzón o caso Bretón ${ }^{8}$ ).

Describir la naturaleza de los hechos que se narran constituye, de entrada, un punto de divergencia, a pesar de que los medios parten de una materia prima informativa común. Así, la huelga general del 29-M se lee, según uno u otro periódico, en términos de fracaso o logro sindical. La adjetivación y el léxico constituyen el principal recurso para sostener las propuestas: "Huelga parcial" [LV], "Huelga poco general" $[\mathrm{ABC}]$, "Derrota sindical" [EM]. Sin embargo, más allá de las nominalizaciones, algunos añaden estrategias de focalización, como los dos puntos: "29-M: día laborable" [LR] -que repite en el primer subtítulo: "La huelga fracasa: los sindicatos consiguen menos seguidores [...]"-; o de contraste: "La gran huelga no mueve a Rajoy" [EC], "Fracasa la huelga, ganan los camorristas" [LG], "La calle aprieta/Rajoy no para" [EpC].

Los tres últimos ejemplos, si bien emplean una estrategia similar con fines opuestos, incorporan, además, otros puntos de encuadre. La Gaceta define a los agentes como "camorristas", un término que retoma en el subtítulo, como "radicales antisistema". En este contexto apuesta por una colocación para subrayar el guión del fracaso: "UGT y Comisiones Obreras elevan cómicamente el paro al 85\%". Por su parte, EpC y EC coinciden en poner la atención en la ausencia de consecuencias, con el uso de la negación, que contrasta con las estrategias para destacar el apoyo social a la protesta en los subtítulos: "Euskadi vive el mayor paro en una década de manifestaciones multitudinarias" [EC] o "La huelga triunfó en sectores clave y las manifestaciones fueron masivas" [EpC]. Otros medios dejan la valoración (éxito/fracaso) para el antetítulo o el subtítulo y se decantan por subrayar los efectos en el titular, con los trabajadores

5 Publicados en las ediciones impresas del 15 de abril, 18 de septiembre, el 22 de octubre y el 26 de noviembre de 2012.

6 Aparecidos en las ediciones del 9 de mayo, 10 de junio y 21 de julio.

7 Recogida en los periódicos del 30 de marzo.

8 En las portadas del 10 de febrero y 28 de agosto, respectivamente.

9 Los fragmentos subrayados ilustran los indicadores a los que se hace referencia. En cursiva se resaltarán otros que sirven para reforzar el punto de vista en esos fragmentos. 
como agente de futuras acciones y distintos grados de intensidad: "Los sindicatos anuncian "conflictividad creciente" " [LVG], "Los sindicatos alertan: Las protestas no han hecho más que empezar" [LE] -en este caso con la fórmula antepuesta y con una locución que le confiere un tono más coloquial-; o "Los sindicatos amenazan a Rajoy con un 'conflicto social creciente" " [EP].

Tampoco se presentan nítidos los términos a la hora de explicar la renuncia a un cargo público, sea en la administración o en una entidad financiera. La dimisión de Rodrigo Rato al frente de Bankia responde, según algunos medios, a una imposición, mientras que para otros implica una decisión personal. El País, ABC, El Mundo y Levante-EMV identifican al Gobierno como agente que "obliga/fuerza la salida/la marcha de Rato", aunque los tres últimos hacen explícita la causa: "para salvar/sanear Bankia". La Gaceta, en cambio, pone nombre a los agentes pero marca distancias respecto al efecto, al elegir un verbo -en pretérito- que no incide en la presión: "Rajoy y de Guindos pidieron a Rato que abandonara la Presidencia de Bankia". Para otros, en cambio, tiene un objetivo casi altruista: "Rato se va para facilitar la salvación de Bankia con el dinero del Estado" [LVG] o "Rato deja Bankia tras anunciar Rajoy que saneará la entidad" [LR]. En un término medio se sitúa La Vanguardia, ya que tras un "Rato se aparta", completa la interpretación en el subtítulo: "El presidente de Bankia tira la toalla tras varios meses de presión del Gobierno". Por su parte, EpC se inclina por una lectura en clave de consecuencias, con el título "Bankia somos todos" y un subtítulo con inciso explicativo: "Economía toma las riendas de la cuarta entidad financiera, que necesitará entre 5.000 y 10.000 millones de las arcas públicas". En la misma línea, El Correo, sin entrar a valorar las circunstancias de la marcha, destaca también este aspecto: "La salida de Rato allana el camino para reflotar Bankia con dinero público".

Así mismo, la marcha de Esperanza Aguirre de la presidencia de la Comunidad de Madrid se presta a varias lecturas, desde las que ponen el acento en el hecho inesperado a las que aluden a que se trata de algo sopesado, al añadir el complemento temporal: "Un adiós meditado desde hace un año" [ABC]. O, como La Razón, optan por una exhortación, aunque sin exclamaciones: "Arriba Esperanza" y un subtítulo que, de nuevo, apela a la temporalidad para despejar otras razones: "La presidenta de Madrid presenta una dimisión que medita desde hace más de un año [...]". Tras una fórmula nominal en el titular -"Adiós enigmático"-, EpC sugiere en los subtítulos circunstancias poco transparentes: "Esperanza Aguirre abandona la presidencia de Madrid sin aclarar los motivos de la decisión", y a las consecuencias que plantea: "El sucesor que nombra es un claro desafío a la línea del PP de Rajoy". En esta misma línea apunta El País: "Esperanza Aguirre dimite tras imponer a su sucesor a Rajoy", con un subtítulo que define al elegido con un inciso entre comas: "Ignacio González, enfrentado a La Moncloa, presidirá la Comunidad". El conflicto es el hilo conductor también para LV, al señalar los efectos: "Aguirre se retira y abre un nuevo frente a Rajoy”, y las condiciones, en el subtítulo: “[...] deja la política por razones personales e impone ella misma a su sucesor". Es interesante destacar las anáforas empleadas por LV para referirse a ella como "Thatcher española" o "una voz discrepante", mientras otras publicaciones eligen un irónico "lideresa" [EpC] o "principal líder [del] sec- 
tor discrepante del PP" [EP]. Tanto EM como LE se centran en la marcha, aunque la lectura varía de forma importante. Si para el primero implica un referente del liberalismo: "La España liberal pierde a su principal referente", los términos bélicos del segundo muestran una visión más crítica de su papel: "La dimisión de Aguirre desarma el ala dura del PP".

Aunque la base de la noticia sea una cifra, las interpretaciones configuran realidades diferentes. La concesión de 100.000 millones de euros por parte del Eurogrupo para recapitalizar los bancos españoles da lugar a un abanico de deducciones que abarcan desde la propia definición del hecho a las circunstancias y consecuencias que plantea. La palabra rescate define, para la gran mayoría, el estado de la cuestión. Sin embargo, la adjetivación o la introducción de otros complementos matizan en buena medida la interpretación. Lo que para alguno es un "Rescate" [LVG] sin más, o un "Rescate a la banca" [LV] y un "Rescate ilimitado a la banca" [LE], para otros va mucho más allá e implica a todo el país, en la línea de El Correo y El País: "Rescate a España”. No obstante, otros medios sitúan la lectura en las antípodas. La Razón opta por términos futbolísticos -coincidiendo con el arranque de la Eurocopa- para concluir que "España despeja el rescate", mientras EM recurre a la nominalización y a un término muy connotado para definir la operación: "Rescate sin humillación", en la línea en la que se posiciona $A B C$, evitando la palabra rescate e incluyendo una apostilla: "Europa financia a la banca sin condiciones para España".

También la iniciativa de la petición muestra variaciones. Lo que para algunos es un logro, para otros representa una claudicación, según reflejan los verbos empleados en los subtítulos o destacados. Así, "El Gobierno logra un préstamo de hasta $100.000 \mathrm{mi}-$ llones para la banca sin condiciones" [LR] choca con opciones como: "España, obligada a pedir ayuda para recapitalizar bancos [...]" [EM]; "Rajoy recurre al fondo de ayuda europeo para sanear la banca" [EP]; "El Gobierno español, forzado por el FMI, acepta una ayuda de hasta 100.000 millones para sanear el sistema financiero" [LE]; o "España pide cien mil millones de euros para salvar la banca" [LVG].

La situación alcanzada por la prima de riesgo el 19 de julio de 2012, si bien no genera demasiadas discrepancias respecto a la gravedad de los hechos -a través de metáforas que remiten al campo de la salud: "España entra en coma" [LG], "Alerta máxima" [EC]; "Al límite" [LVG]-, sí plantea matices sobre la responsabilidad de los actores, mediante fórmulas nominales con valor vocativo: "La hora del BCE" [LR] o "BCE ahora o nunca" $[\mathrm{ABC}]$; o de las posibles consecuencias: "La prima se desboca a 610 puntos y empuja a España al rescate total" [EM]. También varía la identificación de las causas. Lo que para unos responde a la petición valenciana de acogerse al rescate autonómico -"El diferencial rompe la barrera de los 600 tras anunciar la Generalitat Valenciana que se acogía al Fondo Autonómico" [EM] o "La Comunidad Valenciana pide el rescate y dispara la prima de riesgo a 612 puntos" [EP]-, para otros es una estrategia orquestada -"Los especuladores se ceban con España ante la pasividad del Banco Central Europeo" [LR]-, o de desconfianza: "El duro ajuste de Rajoy no devuelve la confianza" [EpC].

Los resultados electorales son un marco dado a las más variadas interpretaciones, más aún si tienen de fondo una apuesta como la de CiU en las autonómicas del $25 \mathrm{~N}$. 
La pérdida de 12 escaños por la formación, tras lanzar su apuesta soberanista y reclamar un respaldo mayoritario, está fuera de duda; sin embargo, el alcance de las valoraciones muestra diferencias importantes y da pie a un catálogo de recursos que van desde el coloquialismo -"Batacazo" [EpC]- a fórmulas apelativas -"Adéu Mas, a Cataluña le gusta España" [LR]-, metáforas -"Duro castigo a Mas" [LV], "El plan de Mas se hunde en las urnas [EP] o "Artur Mas cae al abismo" [LG]- o, incluso, el recurso a la ironía, con un juego entre titular: "Mas entra en la historia", y epígrafe: "Protagoniza el mayor ridículo electoral en 30 años de política autonómica" [EM]. El uso de determinados sustantivos y adjetivos constituye un elemento clave a la hora de matizar: "Espectacular descalabro de CiU con su quimera soberanista" [ABC] -tras titular "Mas pierde su plebiscito"-, "CIU gana pero sufre un durísimo castigo [...]" [EP] o "[CiU] Pierde 12 escaños y queda en manos de un nacionalismo radicalizado" [LR].

Los comicios gallegos y vascos del 21 de octubre plantean un escenario de lecturas más allá de los propios resultados. La intensidad y sentido de la victoria del PP en Galicia puede ser "Aire para Rajoy" [Epc] o un "Aval absoluto para Rajoy" [LR], dos extremos entre los que se sitúan propuestas como "Galicia rescata a Rajoy" [LV], "[...] Feijóo salva a Rajoy" [EM] o "El desplome socialista da aliento a Rajoy" [EP], donde cabe destacar que el énfasis se pone, no en el logro popular, sino en el "desplome" del partido de la oposición. Esta apreciación contrasta con las que recogen en sus subtítulos La Razón: "La mayoría conseguida en Galicia por Feijóo respalda la política anticrisis del PP frente a la izquierda, que intenta deslegitimarla en la calle", y ABC: "Las políticas de austeridad no castigan al PP [...]", tras titular: "Abrumadora victoria de Feijóo". Del mismo modo, la atribución de responsabilidades varía desde "Las urnas castigan a López" [EC] a "Rubalcaba hunde al PSOE" [LR], pasando por "El PSOE de Rubalcaba se derrumba" [EM] o "Rubalcaba se hunde" [LG]. El léxico connotado constituye una de las claves para valorar el éxito ("barrida popular", "Feijóo arrasa") [EM], pero también para referirse a los actores, como en el caso de EH Bildu, a partir de fórmulas anafóricas como "los proetarras", en LR y LG. Ambas publicaciones señalan al respecto que "El País Vasco abre la grieta independentista" [LR] y "Las elecciones gallegas y vascas aceleran la crisis territorial del Estado" [LG].

Si bien las circunstancias que motivaron la nueva operación de cadera del Rey eran conocidas por los medios, su traducción en el principal titular de portada no siempre las refleja. Solo cuatro aluden directamente a la actividad que realizaba, con opciones que apuntan a ella (con el uso de la pasiva) -"El Rey es operado de la cadera tras caerse en un safari en Botsuana" [EP]-; al ocultamiento -"El batacazo del Rey desvela que llevaba 4 días cazando elefantes" [EM]-; o que le sitúan como sujeto: "El Rey se rompe la cadera en una cacería en Botsuana" [LVG] o "El Rey se rompe la cadera en una cacería de elefantes en Botsuana" [LE]. Otros cinco medios añaden este dato en el subtítulo, tras fórmulas más neutras en el titular: "El Rey, operado tras un accidente en Botsuana" [LV], "Tercera operación del Rey en dos años" [EpC], "La fractura de la cadera tendrá al Rey de baja entre mes y medio y dos meses" [LG], "La inoportuna caída del Rey" [EC] o "El año más amargo" [ABC]. Junto a esta opción expresiva, el periódico monárquico incide en la evolución en los subtítulos -"El Rey se recupera de una operación [...]" o "Se reincorporará al despacho en diez días y normalizará su 
agenda en dos meses"-, para rematar con el editorial "El acoso más hipócrita". Más discreta es la respuesta de LR, que tras apostar por otro tema en su página principal, indica: "El Rey, operado de la cadera tras caerse en Botsuana". La alusión a los costes -"Matar a un paquidermo ronda los 37.000 euros" [LE]-; el secretismo del viaje "El presidente Rajoy desconocía que el jefe del Estado estaba en Botsuana [...]" [EM]-; o la relación con accidentes de caza recientes -"Froilán y la bala perdida" [EpC]- influyen en la interpretación de los hechos al poner sobre la mesa nuevos ingredientes para la lectura.

La identificación de restos humanos en la finca de Córdoba donde desaparecieron dos menores abre varias opciones a la hora de identificar al presunto agente. Frente a unos más prudentes, otros realizan una atribución directa: "Bretón asesinó y quemó a sus hijos" $[\mathrm{ABC}]$; con indicios, a través de la anticipación: "Los niños de Córdoba fueron quemados: todo apunta al padre" [LV]; o mediante un giro que, sin citar el nombre, lo deja entrever: "Dos antropólogos desenmascaran al criminal que burló a la policía" [EM] o "Los dientes de leche delatan al asesino" [LR]. En el caso de $L a$ Razón, va más allá del juicio con un segundo titular: "Cadena perpetua", que completa el subtítulo: "Clamor social para que el culpable no salga jamás de la cárcel". La identificación de los hechos dista mucho de la que realiza La Gaceta en su encabezamiento: "Un forense confirma restos humanos en la finca de Bretón" y que asumen $\mathrm{EpC}$, con un titular apelativo: "Giro espectacular en el 'caso Bretón'”, y EP, al remitir a las causas: "Un error policial retrasa un año el caso de los niños Ruth y José". En cambio, La Voz de Galicia va más allá de las evidencias probadas y sin identificar al posible agente, aventura lo sucedido: "Dos informes de antropólogos revelan que los niños Ruth y José fueron incinerados".

Uno de los casos que mayor disparidad de propuestas genera es la sentencia que inhabilitó al juez Baltasar Garzón. Así, mientras en unos casos aparece como una víctima de la justicia -"Garzón sí paga por el 'Gürtel” " [EpC], al reforzar un verbo claramente connotado con un operador argumentativo y subrayar la visión con el epígrafe "Dura condena"-, otros recurren a la lectura inversa: "Garzón $\backslash$ Justicia para el 'justiciero" " [LR], con las que el periódico se distancia respecto a la consideración de Garzón como alguien "que observa y hace observar estrictamente la justicia". La Razón incorpora, además, algunos epígrafes explícitamente connotados para introducir dos de los subtítulos: "La última Garzonada", para referirse a la valoración de la sentencia hecha por Garzón y "Fans antisistema", un término genérico que trata de desvirtuar a quienes apoyan al juez.

$A B C$ y El Mundo llevan al titular una breve alusión de los jueces del Tribunal Supremo, pero significativa por la trayectoria de Garzón en la persecución de delitos contra los derechos humanos. Mientras el primero se decanta por una lectura directa: "Garzón/ Inhabilitado once años por sus métodos totalitarios", el segundo toma distancia, tanto al introducir la expresión comparativa como al utilizar las comillas: "Garzón expulsado por actuar como los jueces de los 'regímenes totalitarios"'. En la línea de EM, también hacen mención de ello en portada La Razón y La Voz de Galicia.

La idea de expulsión sirve como trasfondo a las versiones ofrecidas por seis medios, aunque se adscribe a una escala que va desde la constatación de las consecuen- 
cias del fallo, a través de una contundente y efectista nominalización: "Ex juez Garzón" [LV], a opciones que imprimen un sentido muy connotado, con un inciso entre comas: "Garzón, el juez prevaricador, expulsado de la carrera judicial" [LG]. El resto escoge una estructura en activa que hace recaer la iniciativa de la acción en el órgano judicial, aunque con un tono diferente. Mientras algunos marcan distancias entre el responsable y el afectado, a través de una expresión directa -"El Supremo expulsa a Garzón de la judicatura" [LVG]- o de una perífrasis que amortigua el impacto del verbo -"El Supremo pone fin a la carrera judicial de Garzón" [LE] o "El Tribunal Supremo pone punto final a la carrera judicial de Garzón" [EC]-, El País opta por la personalización, reforzada, si cabe, por la elección de un verbo como acabar y la relación directa con la persona juzgada: "El Supremo acaba con Garzón", y por ende, con todo lo que representa su figura.

\section{Conclusiones}

Los resultados de la investigación ponen de manifiesto la importancia de ampliar el objetivo de análisis más allá de la homogeneización de contenidos o de la coincidencia en la selección de temas de portada, para determinar con mayor precisión el enfoque elegido, el encuadre que imponen los medios a la hora de aprehender una realidad que, inevitablemente, contribuyen a construir.

En primer lugar, la combinación de las aportaciones de la teoría del framing y de la metodología pragmaestilística -con su atención a las estructuras microlingüísticasha permitido captar detalles que pasarían desapercibidos al enfoque genérico del encuadre. En este sentido, como demuestra el estudio, la coincidencia en la atención a unos hechos o agentes, la atribución de causas o consecuencias, no siempre se corresponde con una interpretación equivalente. Así pues, son determinados indicadores lingüísticos (incisos, comillas, nominalizaciones, focalizadores, operadores argumentativos...) los que contribuyen a definir de manera más nítida las fronteras del marco interpretativo.

En segundo lugar, cabe señalar la idoneidad de un estudio comparativo entre distintos medios, ya que puede proporcionar una base sólida para observar cuáles son los aspectos que unos u otros exhiben o silencian, precisamente en un espacio privilegiado donde se condensa la personalidad de la publicación.

En tercer lugar, la formulación sintética de los titulares y su condición de reclamo privilegia la presencia de operadores pragmaestilísticos léxicos, desde sustantivos o verbos a adjetivos -destinados especialmente a identificar los hechos o a los agentes que intervienen-, en los que la interpretación se evidencia de forma más explícita y decantada. En cambio, no son tan frecuentes otros indicadores presentes en el discurso periodístico, vinculados a avanzar en la información o a introducir otras voces. No obstante, las opciones de focalización o los incisos tienen un peso determinante, junto con las apostillas introducidas a través de complementos circunstanciales de tiempo, modo o finalidad.

Finalmente, es importante resaltar la tendencia a la acumulación en la presencia de indicadores, de modo que aquellos titulares que presentan algunos recursos pragmaestilísticos, suelen estar acompañados de subtítulos, epígrafes o destacados con 
otros operadores, en ocasiones con cierta complementariedad. Esta circunstancia es remarcable en tres de los medios analizados: $A B C$, La Razón y La Gaceta, en especial en las informaciones con un componente político, en la sentencia al juez Garzón o, a excepción del tercero, en el caso Bretón.

\section{Referencias}

BARNHURST, Kevin G., NERONE, John (2011): The form of news. A History. New York, Guildford Press.

BISELLI, Rubén (2005): “La portada de La Nación como dispositivo discursivo". La Trama de la Comunicación, vol. 10, pp. 105-116.

BOCZKOWSKI, Pablo J. (2010): News at work. Imitation in an Age of Information Abundance. Chicago, University of Chicago Press.

CONBOY, Martin (2006): Tabloid Britain. Constructing a community through language. London, Roudledge.

COPERÍAS, María José y GÓMEZ MOMPART, Josep Lluís (2013): “The Changing Identity of British Broadsheets", en MATEOS-APARICIO MARTIN-ALBO, Ángel y DE GREGORIO-GODEO, Eduardo (eds.): Culture and Power: Identity and Identification. Newcastle, Cambridge Scholars Publishing, pp. 233-243.

ENTMAN, Robert M. (1993): "Framing: Toward Clarification of a Fractured Paradigm”. Journal of Communication, núm. 43(4), pp. 51-58.

GHANEM, Salma I. (1997): "Segundo nivel de composición de la agenda: la opinión pública y la cobertura del crimen”. Comunicación y Sociedad, vol. X, núm. 1, p. 151-167.

GOFFMAN, Erving (2006): Frame analysis: los marcos de la experiencia. Madrid, Centro de Investigaciones Sociológicas.

HICKEY, Leo (1997): Curso de pragmaestilística. Madrid, Coloquio.

HUBÉ, Nicolas (2010): “«La forme, c’est le fond». La «Une» comme outil de marketing de «modernisation» de la press quotidienne". Questions de Communication, núm. 17, pp. 253-272.

HUMANES, María Luisa e IGARTUA, Juan José (2004): “El encuadre noticioso de la realidad. Reflexiones teórico-metodológicas sobre el concepto de framing". Quaderns de Filologia. Estudis de Comunicació, núm. II, pp. 201-218.

KIM, Sei-Hill, SCHEUFELE, Dietram A. y SHANAHAN, James (2002): "Think about it this way: Attribute agenda-setting function of the press and the public's evaluation of a local issue". Journalism and Mass Communication Quarterly, núm. 79, pp. 7-25.

McCOMBS, Maxwell E. y SHAW, Donald L. (1972): “The agenda-Setting Function of Mass Media". The public opinion Quaterly, vol. 36, núm. 2, pp. 176-187.

MOUILLAUD, Maurice (2002): “O título e os títulos”, en DAYRELL, Sérgio (org.): O jornal. Da forma ao sentido. Brasília, Universidade de Brasilia, pp. 99-116. 
MOUILLAUD, Maurice (2002): "Da forma ao sentido", en DAYRELL, Sérgio (org.): O jornal. Da forma ao sentido. Brasília, Universidade de Brasilia, pp. 29-36.

MURTHY, C. S. H. N., RAMAKRISHNA, Challa \& MELKOTE, Srinivas R. (2010): "Trends in first page priorities of Indian print media reporting. A content analysis of four English Language newspapers". Journal Media and Communication Studies, vol. 2(2), pp. 39-53.

PALAU, Dolors (2008): Estudi pragmaestilístic de la premsa escrita diària. Trets $i$ usos estilístics en les distintes modalitats genèriques dels diaris d'informació general, Tesis doctoral, Universitat Autònoma de Barcelona, Bellaterra, en: http://www.tesisenred.net/bitstream/handle/10803/4209/dps1de1.pdf.

RODRIGO, Miquel (2001): Teorías de la comunicación. Ámbitos, métodos y perspectivas. Bellaterra, Universitat Autònoma de Barcelona. Servei de Publicacions.

SÁDABA, María Teresa (2001): "Origen, aplicación y límites de la 'teoría del encuadre' (framing) en comunicación”, Comunicación y sociedad, vol XIV, núm. 2, pp. 143-175.

SCHEUFELE, Dientram A. (1999): "Framing as a theory of media effects". Journal of Communication, num. 49, pp.103-122.

VREESE, Claes H. (2005): "News framing: Theory and typology". Information Design Journal+Document Design, num. 13 (1), pp. 51-62. 